TITLE:

\title{
High resolution numerical relativity simulations for the merger of binary magnetized neutron stars
}

\section{$\operatorname{AUTHOR}(\mathrm{S})$ :}

Kiuchi, Kenta; Kyutoku, Koutarou; Sekiguchi, Yuichiro; Shibata, Masaru; Wada, Tomohide

\section{CITATION:}

Kiuchi, Kenta ...[et al]. High resolution numerical relativity simulations for the merger of binary magnetized neutron stars. Physical Review D 2014, 90(4): 041502.

\section{ISSUE DATE:}

2014-08-28

URL:

http://hdl.handle.net/2433/189621

RIGHT:

(C) 2014 American Physical Society 
PHYSICAL REVIEW D 90, 041502(R) (2014)

\title{
High resolution numerical relativity simulations for the merger of binary magnetized neutron stars
}

\author{
Kenta Kiuchi, ${ }^{1}$ Koutarou Kyutoku, ${ }^{2}$ Yuichiro Sekiguchi, ${ }^{1}$ Masaru Shibata, ${ }^{1}$ and Tomohide Wada ${ }^{3}$ \\ ${ }^{1}$ Yukawa Institute for Theoretical Physics, Kyoto University, Kyoto 606-8502, Japan \\ ${ }^{2}$ Department of Physics, University of Wisconsin-Milwaukee, P.O. Box 413, Milwaukee, \\ Wisconsin 53201, USA \\ ${ }^{3}$ National Astronomical Observatory of Japan, Mitaka 181-8588, Japan \\ (Received 22 April 2014; published 28 August 2014)
}

\begin{abstract}
We perform high-resolution magnetohydrodynamics simulations of binary neutron star mergers in numerical relativity on the Japanese supercomputer $\mathrm{K}$. The neutron stars and merger remnants are covered by a grid spacing of $70 \mathrm{~m}$, which yields the highest-resolution results among those derived so far. By an in-depth resolution study, we clarify several amplification mechanisms of magnetic fields during the binary neutron star merger for the first time. First, the Kelvin-Helmholtz instability developed in the shear layer at the onset of the merger significantly amplifies the magnetic fields. A hypermassive neutron star (HMNS) formed after the merger is then subject to the nonaxisymmetric magnetorotational instability, which amplifies the magnetic field in the HMNS. These two amplification mechanisms cannot be found with insufficient-resolution runs. We also show that the HMNS eventually collapses to a black hole surrounded by an accretion torus which is strongly magnetized at birth.
\end{abstract}

\section{INTRODUCTION}

Coalescence of binary neutron stars (BNS) is one of the most promising sources of gravitational waves. The secondgeneration gravitational-wave detectors like advanced LIGO, advanced VIRGO, and KAGRA [1], which will operate in a few years, may detect gravitational waves from BNS mergers as frequently as $\sim 1-100 / y r$ [2,3]. If gravitational waves from BNS mergers are observed, they could tell us the validity of general relativity in strong gravitationalfield regions and the equation of state (EOS) of neutron stars. Furthermore, a long-standing puzzle on the central engine of short-hard gamma-ray bursts (SGRB) may be resolved if gravitational waves are observed simultaneously with them. BNS also attracts attention as a possible site of r-process nucleosynthesis [4] and as a source of electromagnetic transients. In particular, emission associated with the radioactive decay of the r-process elements in the merger ejecta is a promising electromagnetic counterpart of BNS mergers [5]. In fact, "kilonova" associated with GRB130603B is an interesting candidate of such events [6]. All these facts stimulate us to theoretically construct a reliable model of the BNS merger. Numerical relativity is the unique approach for this purpose.

Strong magnetic fields are universal elements of neutron stars, shown by pulsar observations [7]. Typical strength of the magnetic fields is $10^{11}-10^{15} \mathrm{G}$. The so-called magnetars have even stronger magnetic fields of $10^{14}-10^{15} \mathrm{G}$. Although magnetic fields could be a key ingredient in the BNS mergers, their role is still not clear. The prime reason is that a number of magnetohydrodynamical instabilities, which can amplify the magnetic fields, are generally activated by short-wavelength modes, i.e., the fastest growing mode has a short wavelength and is not easily resolved in numerical simulations. One example is the Kelvin-Helmholtz (KH) instability. In the absence of gravity, this instability sets in for all the wavelengths, and moreover, the shorter-wavelength modes have the larger growth rates. Another example is the magnetorotational instability (MRI) [8], in which the wavelength of the fastest growing mode is quite short for the typical magnetic-field strength and density of neutron stars. It has not been easy to prepare a sufficient grid resolution for the BNS merger simulations for them [9].

We tackle this problem using the 10 PFLOPS Japanese supercomputer $\mathrm{K}$, which enables us to assign the highest grid resolution so far in this field. To assess the resolution dependence of the magnetic-field amplification processes, we carry out an in-depth resolution study. Furthermore, to explore the final state of the BNS merger, we perform long term simulations of duration $\sim 100 \mathrm{~ms}$. Together with the recent observations of $\approx 2 M_{\odot}$ neutron stars [10], the recent numerical relativity simulations have established that, in the BNS mergers for the typical total mass $2.6-2.8 M_{\odot}$ and for plausible EOS, a hypermassive neutron star (HMNS) is transiently formed after the merger and subsequently it collapses to a black hole [11]. Based on this picture, we focus in particular on the following three stages. First is the stage in which two neutron stars come into contact. This stage is subject to the $\mathrm{KH}$ instability, which develops in a thin shear layer [12]. The second is the HMNS phase which is subject to the MRI because of a rapid and strong differential rotation [13]. The third is the stage after the HMNS collapses to a black hole (BH) surrounded by an accretion torus, which could be again subject to the MRI. 
Throughout the analysis for the three stages, we clarify the amplification mechanisms of magnetic fields.

\section{METHOD, INITIAL MODELS AND GRID SETUP}

Einstein's equation is solved in the puncture-BSSN formalism [14]. The magnetohydrodynamics (MHD) equation is solved by a high-resolution shock-capturing scheme with the third-order cell reconstruction (see Ref. [15] for details). A fixed mesh-refinement algorithm is employed to resolve the wide dynamical range of BNS mergers simultaneously, where we prepare 7 refinement levels with the varying grid spacing as $\Delta x_{l}=2^{7-l} \Delta x_{7}(l=1,2, \ldots, 7)$ for the same coordinate origin. Here, $\Delta x_{l}$ is the grid spacing for the $l$ th level in the Cartesian coordinates. The solenoidal constraint and magnetic flux conservation on the refinement boundary are satisfied using the Balsara's method $[15,16]$. The orbital plane symmetry is imposed. For each level, the computational domain covers $\left[-N \Delta x_{l}, N \Delta x_{l}\right]$ for $x$ and $y$ directions, and $\left[0, N \Delta x_{l}\right]$ for $z$ direction. The highest-resolution runs were done with 16,384 CPUs on the $\mathrm{K}$.

Table I lists the key parameters of our models and numerical setup of the simulations. We employ H4 EOS [17], with which the maximum mass of neutron stars is $2.03 M_{\odot}$, and the mass of each neutron star is chosen to be $1.4 M_{\odot}$. With the parameters chosen, the computation follows about 6 inspiral orbits, and the merger outcome is a HMNS with its lifetime $\sim 10 \mathrm{~ms}$ in the absence of magnetic fields [11]. We prepare three grid resolutions with $\Delta x_{7}=70,110$, and $150 \mathrm{~m}$ as well as three maximum initial magnetic-field strengths, $10^{14.5}, 10^{15}$, and $10^{16} \mathrm{G}$ to assess how the result depends on the resolution and field strength. The initial magnetic field is given in terms of the vector potential

$$
A_{i}=\left(-\left(y-y_{\mathrm{c}}\right) \delta_{i}^{x}+\left(x-x_{\mathrm{c}}\right) \delta_{i}^{y}\right) A_{\mathrm{b}}\left[\max \left(P-P_{\mathrm{c}}, 0\right)\right]^{2},
$$

TABLE I. Parameters of the BNS and grid setup. $\Delta x_{7}$ is the grid spacing in the finest refinement level and $N$ is the grid number in one positive Cartesian direction. The last column is the initial maximum strength of the magnetic field. Model name follows the EOS, the initial maximum field strength, and grid spacing. The sum of the ADM masses in isolation of each NS $\left(m_{0}\right)$ is $2.8 M_{\odot}$ in all the models. The initial orbital angular velocity $\Omega$ is set to be $G m_{0} \Omega / c^{3}=0.0221$ in all the models with $G$ and $c$ being the gravitational constant and the speed of light, respectively.

\begin{tabular}{lccc}
\hline \hline Model & $\Delta x_{7}[\mathrm{~m}]$ & $N$ & $\log _{10}\left[\mathrm{~B}_{\max }(\mathrm{G})\right]$ \\
\hline H4B15d70 & 70 & 512 & 15.00 \\
H4B15d10 & 110 & 322 & 15.00 \\
H4B15d150 & 150 & 240 & 15.00 \\
H4B14d70 & 70 & 512 & 14.52 \\
H4B16d70 & 70 & 512 & 16.00 \\
H4B16d110 & 110 & 322 & 16.00 \\
H4B16d150 & 150 & 240 & 16.00 \\
\hline \hline
\end{tabular}

where $x_{\mathrm{c}}$ and $y_{\mathrm{c}}$ are the coordinates of the stellar centers, $P$ is the pressure, and $P_{\mathrm{c}}$ is the pressure for $\rho=0.04 \rho_{\max } . A_{\mathrm{b}}$ determines the field strength. The EOS is parametrized by a piecewise polytrope [18] and the $\Gamma$-law EOS is added during the simulation to take into account the shock heating effect with the gamma index being 1.8 (see [11] for details).

\section{RESULTS}

Figure 1 plots the profiles of the density, magnetic-field strength, and magnetic-field lines at selected time slices for H4B15d70 [19]. The magnetic fields do not affect the inspiral dynamics because the magnetic stress energy is much smaller than the matter pressure [9]. The left panel shows a snapshot just after two neutron stars come into contact. In this phase, the $\mathrm{KH}$ vortices develop and curl the magnetic-field lines, generating the strong toroidal fields. This significantly enhances the magnetic stress energy in the shear layer. The unstable shear layer disappears in a dynamical time scale of $\sim 0.1 \mathrm{~ms}$, because the compression and resulting shock heating associated with violent oscillations of the formed HMNS suppress the continuous generation of the vortices.

The middle panel plots a snapshot in the HMNS phase. This shows that large-scale toroidal magnetic fields, enhanced primarily by magnetic winding, are generated. Furthermore, a detailed analysis elucidates that the magnetic fields are also globally amplified by the MRI (see below). The HMNS collapses to a BH at $\approx 14 \mathrm{~ms}$ after the merger and a part of the HMNS forms an accretion torus surrounding the $\mathrm{BH}$. The nondimensional $\mathrm{BH}$ spin is $\approx 0.69$ and the torus mass is $\approx 0.06 M_{\odot}$ at $10 \mathrm{~ms}$ after the $\mathrm{BH}$ formation for H4B15d70. These numbers depend slightly on the grid resolution.

The MRI preserves the turbulent flow and vortices inside the accretion torus and they enhance the accretion due to the outward angular-momentum transport. The density of the accretion torus gradually decreases and $10^{10}-10^{11} \mathrm{~g} / \mathrm{cm}^{3}$ for $\sim 10-30 \mathrm{~ms}$ after the BH formation. The magnetic field still remains to be toroidal-field dominant, and we do not find any coherent poloidal field at this moment as shown in the right panel of Fig. 1. This is in contrast to the result of Ref. [20], which reported the formation of a coherent poloidal field within a relatively short timescale, i.e., $\approx 12 \mathrm{~ms}$ after the $\mathrm{BH}$ formation. ${ }^{1}$ It is not trivial to generate such a coherent poloidal field. A large amount of matter is ejected and blown outwards in the merger phase and the resulting ram pressure due to the fall-back toward the $\mathrm{BH}$ and torus suppresses the matter outflow. Since the magnetic-field lines are frozen in the fluid elements, an outflow which has not been seen for $t-t_{\mathrm{mrg}} \lesssim 40 \mathrm{~ms}$ will be necessary to generate a coherent poloidal magnetic field.

\footnotetext{
${ }^{1}$ The coherent poloidal field structure is probably due to the choice of seeds [21].
} 

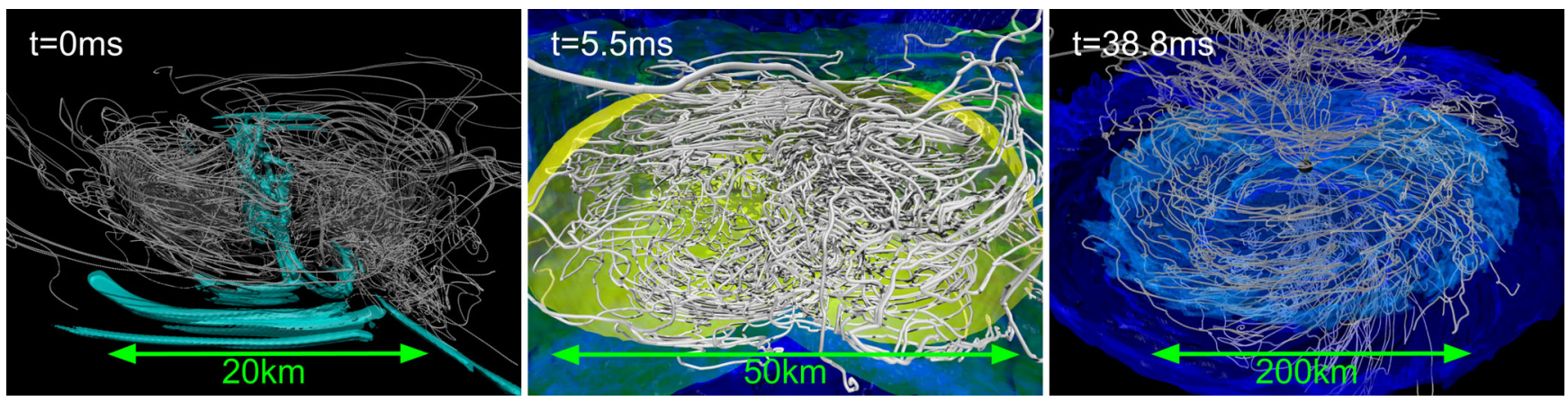

FIG. 1 (color online). Snapshots of the density, magnetic-field strength and magnetic-field lines for $\mathrm{H} 4 \mathrm{~B} 15 \mathrm{~d} 70$ at $t-t_{\mathrm{mrg}} \approx 0.0 \mathrm{~ms}$ (left panel), at $t-t_{\mathrm{mrg}} \approx 5.5 \mathrm{~ms}$ (middle panel), and at $t-t_{\mathrm{mrg}} \approx 38.8 \mathrm{~ms}$ (right panel). $t_{\mathrm{mrg}}$ is a time when the amplitude of the gravitational waves becomes maximum. The left, middle, and right panels show the configuration just after the onset of the merger, for the HMNS phase, and for a BH surrounded by an accretion torus, respectively. In each panel, the white curves are the magnetic-field lines. In the left panel, the cyan represents the magnetic fields stronger than $10^{15.6} \mathrm{G}$. In the middle panel, the yellow, green, and dark blue represent the density iso-surface of $10^{14}, 10^{12}$, and $10^{10} \mathrm{~g} / \mathrm{cm}^{3}$, respectively. In the right panel, the light and dark blue are the density iso-surface of $10^{10.5}$ and $10^{10} \mathrm{~g} / \mathrm{cm}^{3}$, respectively.

Figure 2 plots the magnetic-field energy as a function of time for H4B15 runs, H4B14d70, and H4B16d70. Soon after the onset of the merger, the magnetic-field energy is steeply amplified because the $\mathrm{KH}$ vortices develop in
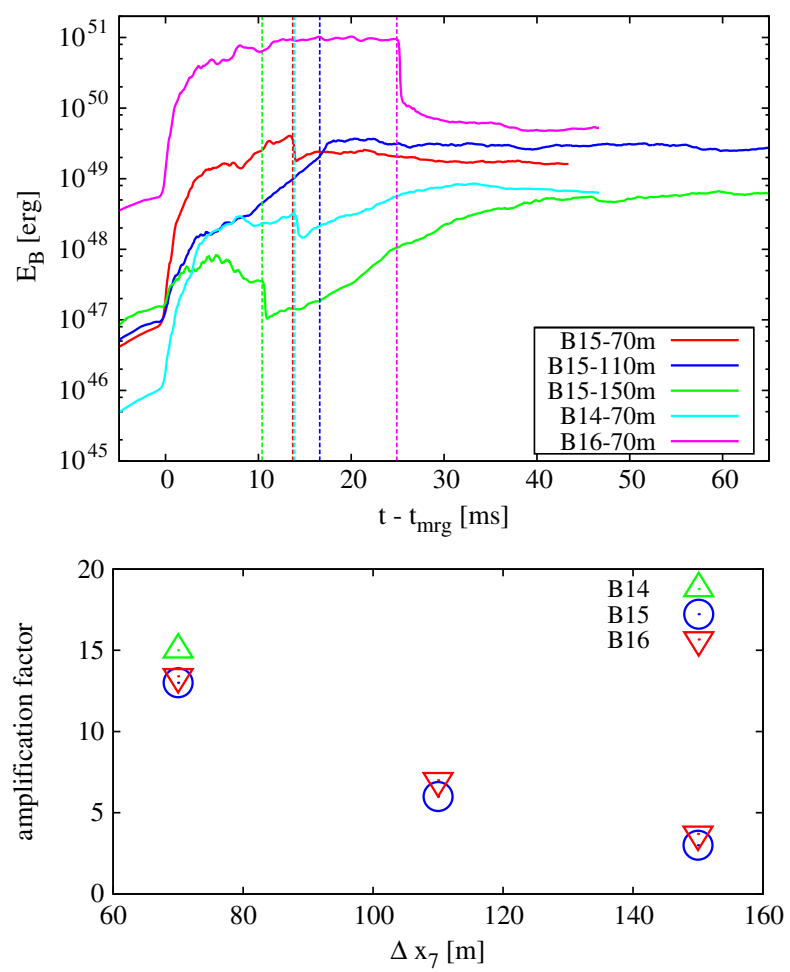

FIG. 2 (color online). (Top) The total magnetic-field energies as a function of time for H4B15 runs with three grid resolutions (B15-70m, B15-110m, B15-150m), for H4B14d70 (B14-70m), and for H4B16d70 (B16-70m). The thin vertical lines denote the formation time of the $\mathrm{BH}$. $\mathrm{E}_{\mathrm{B}}$ is calculated by a volume integral only outside the BH horizon. (Bottom) The dependence of the amplification factor of the maximum toroidal magnetic field in the merger on the grid resolution for all the models. the shear layer. The growth rate is higher for the higherresolution runs, because the growth rate of the $\mathrm{KH}$ instability is proportional to the wave number and hence the smaller-scale vortices have the larger growth rate. We analyze the maximum magnetic-field strength and plot the amplification factor in the merger as a function of $\Delta x_{7}$ in the lower panel of Fig. 2. This clearly shows that the amplification factor depends on the grid resolution but not on the initial magnetic-field strength. This is consistent with the amplification mechanism due to the $\mathrm{KH}$ vortices and qualitatively consistent with the local shearing-box simulation in Ref. [22]. The magnetic-field energy at $t-t_{\mathrm{mrg}} \approx 5 \mathrm{~ms}$ in the high-resolution run is $40-50$ times as large as that of the low-resolution run.

In the HMNS stage, the magnetic-field strength grows significantly in the high- and middle-resolution runs but not in the low-resolution run. We analyze the field amplification by foliating the HMNS in terms of the rest-mass density, i.e., calculating the magnetic-field energy for $\rho_{1} \leq$ $\rho \leq \rho_{2}$ varying $\rho_{1}$ and $\rho_{2}$. The left panel of Fig. 3 plots magnetic-field energy of a radial component for H4B15 runs with $\rho_{1}=10^{11} \mathrm{~g} / \mathrm{cm}^{3}$ and $\rho_{2}=10^{12} \mathrm{~g} / \mathrm{cm}^{3}$. We find that it grows in the middle- and high-resolution runs but not significantly in the low-resolution run. We also find the high- and middle-resolution runs satisfy the criterion $\lambda_{\mathrm{MRI}}^{\varphi} / \Delta x_{7} \geq 10$ where $\lambda_{\text {MRI }}^{\varphi}$ is the MRI wavelength of the fastest growing mode for the toroidal magnetic field, whereas the low-resolution run does not satisfy this criterion.

We fit the growth rate of the magnetic-field energy by $\propto \mathrm{e}^{2 \sigma\left(t-t_{\text {mrg }}\right)}$ for $8 \lesssim t-t_{\text {mrg }} \lesssim 14 \mathrm{~ms}$ for the high-resolution run and find that $\sigma \approx 140 \mathrm{~Hz}$ (for the middle-resolution run, it is $\approx 130 \mathrm{~Hz}$ for $8 \lesssim t-t_{\mathrm{mrg}} \lesssim 16 \mathrm{~ms}$ ) which is several percents of the rotational frequency. This frequency agrees approximately with that of the nonaxisymmetric MRI [23]. The right panel of Fig. 3 plots the magnetic-field energy 

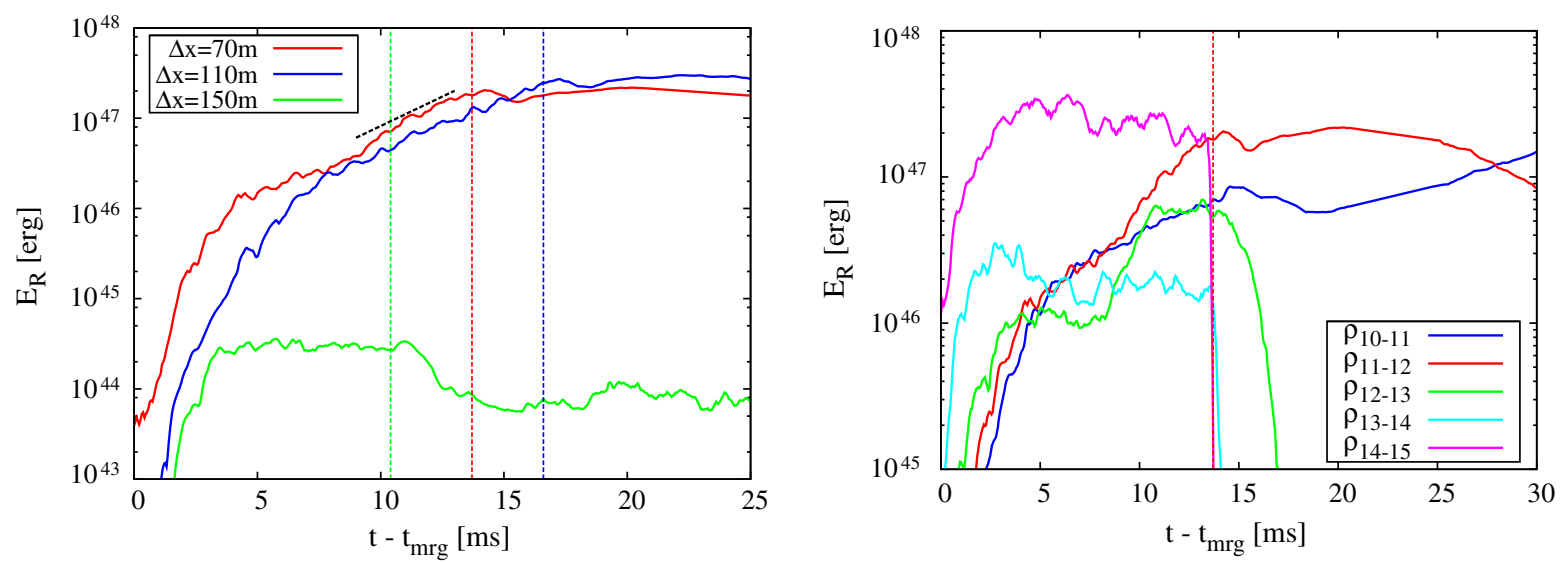

FIG. 3 (color online). (Left) The magnetic-field energy of a radial component in the range $10^{11} \mathrm{~g} / \mathrm{cm}^{3} \leq \rho \leq 10^{12} \mathrm{~g} / \mathrm{cm}^{3}$ for H4B15 runs. The thin vertical lines show the $\mathrm{BH}$ formation time. The black-dashed line is an exponential function $\propto \mathrm{e}^{2 \sigma\left(t-t_{\mathrm{mrg}}\right)}$ with $\sigma \approx 140 \mathrm{~Hz}$ (see text in details). (Right) The magnetic-field energy of the radial component in $10^{a} \mathrm{~g} / \mathrm{cm}^{3} \leq \rho \leq 10^{a+1} \mathrm{~g} / \mathrm{cm}^{3}$ for H4B15d70 with $a=10,11,12,13$, and 14 . The thin vertical line is the $\mathrm{BH}$ formation time.

in various density ranges for $\mathrm{H} 4 \mathrm{~B} 15 \mathrm{~d} 70$. This figure shows that the magnetic field in a high-density region $\rho \gtrsim 10^{13} \mathrm{~g} / \mathrm{cm}^{3}$ does not exhibit the significant growth contrary to that in the lower-density region shown in the left panel. This is because the MRI wavelength is proportional to $\rho^{-1 / 2}$, and hence, the wavelength in the high-density region $\left(\rho \gtrsim 10^{13} \mathrm{~g} / \mathrm{cm}^{3}\right)$ is too short to be resolved even in our highest-resolution run. The growth rate in the range $10^{11} \lesssim \rho \lesssim 10^{12} \mathrm{~g} / \mathrm{cm}^{3}$ is greater than that in the range $10^{10} \lesssim \rho \lesssim 10^{11} \mathrm{~g} / \mathrm{cm}^{3}$ for $8 \lesssim t-t_{\mathrm{mrg}} \lesssim 14 \mathrm{~ms}$ because the orbital angular velocity is larger in the higher density region. The same analysis shows that the magnetic fields are amplified even in $10^{13} \mathrm{~g} / \mathrm{cm}^{3} \lesssim \rho \lesssim 10^{14} \mathrm{~g} / \mathrm{cm}^{3}$ for $\mathrm{H} 4 \mathrm{~B} 16 \mathrm{~d} 70$ and not in $10^{12} \mathrm{~g} / \mathrm{cm}^{3} \lesssim \rho \lesssim 10^{13} \mathrm{~g} / \mathrm{cm}^{3}$ for H4B14d70. We conclude that the growth of the magneticfield energy in the HMNS phase is attributed to the nonaxisymmetric MRI in the low-density region. The magnetic winding contributes to the growth of the toroidal magnetic-field energy as well.

The MRI in the HMNS phase greatly amplify the magnetic fields. At the $\mathrm{BH}$ formation, the magnetic-field strength is already saturated in the high- and middleresolution runs as found in Fig. 2, and thus, it does not much increase in the accretion torus formed after the HMNS collapses. On the other hand, the magnetic field is still amplified in the accretion torus in the low-resolution run. This is attributed to the insufficient resolution to capture the MRI in the merger and HMNS phases. Previous simulations often reported this picture due to the insufficient resolution. However, the picture we show in this paper is qualitatively different from it. The growth of the magnetic-field energy inside the accretion torus is also seen for the low magnetic-field model H4B14d70 in Fig. 2 because the wavelength of the fastest growing mode of the MRI in the HMNS is rather short in this model. On the other hand, the magnetic-field energy for H4B16d70 saturates at the formation of the torus in Fig. 2. In reality, the magnetic-field energy may reach the equipartition to the kinetic energy at the merger and inside the HMNS.

\section{SUMMARY AND DISCUSSION}

We have reported the results of long term and highresolution MHD simulations of the BNS merger performed in numerical relativity on the $\mathrm{K}$. The grid resolution employed is highest among the simulations carried out so far.

We have found the $\mathrm{KH}$ vortices, which develop in the shear layer at the onset of the merger, significantly amplify the magnetic-field strength in a dynamical timescale. This feature can be found only by a simulation with the grid spacing of $\lesssim 100 \mathrm{~m}$.

After the formation of a HMNS as a remnant of the merger, the MRI amplifies the magnetic fields in the HMNS. Because the toroidal magnetic fields are dominant in the HMNS, nonaxisymmetric MRI plays a central role in amplifying the magnetic-field strength in this phase.

The HMNS eventually collapses to a BH surrounded by an accretion torus after the substantial angular-momentum transport inside it. Due to the amplification mechanisms discussed above, the accretion torus formed is strongly magnetized even at its formation. The magnetic-field energy is already saturated, and hence, does not exhibit any remarkable growth. This indicates that a central engine of SGRBs would be modeled by a magnetized accretion torus with saturated strength.

Even after the long term evolution, the global structure of the magnetic field is toroidal-field dominant, and any coherent structure of the poloidal component is not found. This does not agree with the previous finding [20]. Our results indicate that the coherent poloidal field is not likely to be generated in several $10 \mathrm{~ms}$ after the $\mathrm{BH}$ formation, 
HIGH RESOLUTION NUMERICAL RELATIVITY ...

because the ram pressure of the fall-back fluid elements toward the $\mathrm{BH}$ and torus is quite strong and hence the outflow motion, which is necessary to generate the poloidal component, is suppressed. This implies that a new mechanism, which enhances the poloidal motion, is necessary.

\section{ACKNOWLEDGMENTS}

We thank K. Nitadori and T. Ishiyama for the optimization on K. Numerical computations were performed on
PHYSICAL REVIEW D 90, 041502(R) (2014)

the supercomputer $\mathrm{K}$ at AICS, XC30 at CfCA of NAOJ, FX10 at Information Technology Center of Tokyo University, and SR16000 at YITP of Kyoto University. This work was supported by Grant-in-Aid for Scientific Research (24244028, 25103510, 25105508, 24740163, 25103512, 23740160), for Scientific Research on Innovative Area (24103001), by HPCI Strategic Program of Japanese MEXT (hp130025, 140211). K. Kyutoku is supported by JSPS Postdoctoral Fellowship for Research Abroad.
[1] J. Abadie et al., Nucl. Instrum. Methods Phys. Res., Sect. A 624, 223 (2010); T. Accadia et al., Classical Quantum Gravity 28, 025005 (2011); 28, 079501(E) (2011); K. Kuroda, Classical Quantum Gravity 27, 084004 (2010).

[2] V. Kalogera, K. Belczynski, C. Kim, R. oshaughnessy, and B. Willems, Phys. Rep. 442, 75 (2007).

[3] J. Abadie et al. (The LIGO Scientific Collaboration and Virgo Collaboration), Classical Quantum Gravity 27, 173001 (2010).

[4] J. M. Lattimer and D. N. Schramm, Astrophys. J. 192, L145 (1974).

[5] L.-X. Li and B. Paczynski, Astrophys. J. 507, L59 (1998); D. Kasen, N. R. Badnell, and J. Barnes, Astrophys. J. 774, 25 (2013); J. Barnes and D. Kasen, Astrophys. J. 775, 18 (2013); M. Tanaka and K. Hotokezaka, Astrophys. J. 775, 113 (2013).

[6] N. R. Tanvir, A. J. Levan, A. S. Fruchter, J. Hjorth, R. A. Hounsell, K. Wiersema, and R. L. Tunnicliffe Nature (London) 500, 547 (2013); E. Berger, W. Fong, and R. Chornock, Astrophys. J. 774, L23 (2013).

[7] R. N. Manchester, G. B. Hobbs, A. Teoh, and M. Hobbs, Astron. J. 129, 1993 (2005).

[8] S. A. Balbus and J. F. Hawley, Astrophys. J. 376, 214 (1991).

[9] Y. T. Liu, S. L. Shapiro, Z. B. Etienne, and K. Taniguchi, Phys. Rev. D 78, 024012 (2008); M. Anderson, E. Hirschmann, L. Lehner, S. Liebling, P. Motl, D. Neilsen, C. Palenzuela, and J. Tohline, Phys. Rev. Lett. 100, 191101 (2008); B. Giacomazzo, L. Rezzolla, and L. Baiotti, Phys. Rev. D 83, 044014 (2011).

[10] P. Demorest, T. Pennucci, S. M. Ransom, M. S. E. Roberts, and J. W. T. Hessels, Nature (London) 467, 1081 (2010); J. Antoniadis et al., Science 340, 1233232 (2013).
[11] K. Hotokezaka, K. Kiuchi, K. Kyutoku, T. Muranushi, Yu-i. Sekiguchi, M. Shibata, and K. Taniguchi, Phys. Rev. D 88, 044026 (2013).

[12] F. Rasio and S. L. Shapiro, Classical Quantum Gravity 16, R1 (1999); D. Price and S. Rosswog, Science 312, 719 (2006).

[13] M. D. Duez, Y. T. Liu, S. L. Shapiro, M. Shibata, and B. C. Stephens, Phys. Rev. Lett. 96, 031101 (2006); M. Shibata, M. D. Duez, Y. T. Liu, S. L. Shapiro, and B. C. Stephens, Phys. Rev. Lett. 96, 031102 (2006); D. M. Siegel, R. Ciolfi, A. I. Harte, and L. Rezzolla, Phys. Rev. D 87, 121302 (2013).

[14] M. Shibata and T. Nakamura, Phys. Rev. D 52, 5428 (1995); T. W. Baumgarte and S. L. Shapiro, Phys. Rev. D 59, 024007(1999); M. Campanelli, C. O. Lousto, P. Marronetti, and Y. Zlochower, Phys. Rev. Lett. 96, 111101 (2006); J. G. Baker, J. Centrella, Dae-Il Choi, M. Koppitz, and J. van Meter, Phys. Rev. Lett. 96, 111102 (2006).

[15] K. Kiuchi, K. Kyutoku, and M. Shibata, Phys. Rev. D 86, 064008 (2012).

[16] D. Balsara, J. Comput. Phys. 174, 614 (2001).

[17] N. K. Glendenning and S. A. Moszkowski, Phys. Rev. Lett. 67, 2414 (1991).

[18] J. S. Read, B. Lackey, B. Owen, and J. Friedman, Phys. Rev. D 79, 124032 (2009).

[19] See the visualization results in http://www2.yukawa .kyoto-u.ac.jp/ kenta.kiuchi/GWRC/.

[20] L. Rezzolla, B. Giacomazzo, L. Baiotti, J. Granot, C. Kouveliotou, and M. A. Aloy, Astrophys. J. 732, L6 (2011).

[21] L. Rezzolla (private communication).

[22] M. Obergaulinger, M. A. Aloy, and E. Müller, Astron. Astrophys. 515, A30 (2010).

[23] S. A. Balbus and J. F. Hawley, Astrophys. J. 400, 610 (1992). 